\title{
Cache Design for Eliminating the Address Translation Bottleneck and Reducing the Tag Area Cost
}

\author{
Yen-Jen Chang†, Feipei Lai†* and Shanq-Jang Ruan* \\ $\uparrow$ Department of Computer Science and Information Engineering \\ *Department of Electrical Engineering \\ National Taiwan University, Taipei, Taiwan \\ \{ychang,flai,stj\}@orchid.ee.ntu.edu.tw
}

\begin{abstract}
For the physical caches, the address translation delay can be partially masked, but it is hard to avoid completely. In this paper, we propose a cache partition architecture, called paged cache, which can not only mask the address translation delay completely but also reduce the tag area dramatically. In the paged cache, we divide the entire cache into a set of partitions, and each partition is dedicated to only one page cached in the TLB. By restricting the range in which the cached block can be placed, we can eliminate the total or partial tag depending on the partition size. In addition, because the paged cache can be accessed without waiting for the generation of physical address, i.e., the paged cache and the TLB are accessed in parallel, the extended cache access time can be reduced largely. We use SimpleScalar to simulate the SPEC2000 benchmarks and perform the HSPICE simulations (with a $0.18 \mu \mathrm{m}$ technology and $1.8 \mathrm{~V}$ voltage supply) to evaluate the proposed architecture. Experimental results show that the paged cache is very effective in reducing tag area of the on-chip L1 caches, while the average extended cache access time can be improved dramatically.
\end{abstract}

\section{Introduction}

Since an on-chip cache can effectively reduce the speed gap between processor and main memory, almost modern processors employ it to boost the system performance. In most processor designs, the cache access is on the critical path of the execution pipeline, and hence determines the cycle time of processor. However, the cache access is further complicated by the use of virtual memory architecture. In a system with virtual memory, the CPU issues virtual addresses, which must be translated into physical addresses before accessing the physical memory. Usually, the translation lookaside buffer (TLB) is used to speed up this address translation. There are two alternative time points for processing the address translation. Depending on whether the cache is physical or virtual, the TLB access can occur before or after the cache access.
If we use the virtual cache, the TLB translation delay can be completely eliminated and removed from the critical path of processor. Unfortunately, due to the synonyms problem [1], despite the apparent performance gain from virtual cache, the hidden cost of this approach is potentially more than its benefits. Consequently, the physical cache is actually much simpler and should be the design of choice if the address translation latency can be masked such that it would not compromise the cycle time. The TLB translation delay can often be partially overlapped, but it is hard to avoid completely [2][3][4] [5][6].

In this paper, we investigate the physical cache architecture from two different perspectives: tag area and system cycle time. To reduce the area cost of implementing the tag array, we took a closer look at the relationship between the TLB and the cache. As we know, in most conventional architecture with physical cache, the cache access follows the TLB access. The cache hit or miss is independent of the result of TLB access. In contrast, we propose a new cache architecture, called paged cache, in which the cache memory must hold only blocks belonging to the pages that are contained in the TLB. We further divide the entire cache into a set of partitions. All partitions have the same size and the number of partitions is the same as the number of TLB entries, i.e., the number of pages cached in TLB. Each partition is dedicated to one and only one page. By restricting the range in which the cached block can be placed, we can eliminate the total or partial tag depending on the partition size. In addition, because the paged cache can be accessed without waiting for the generation of physical address (i.e., the paged cache and the TLB are accessed in parallel), the cache access time can be reduced largely without address translation delay.

The rest of this paper is organized as follows. Section 2 identifies the problems of the conventional cache architecture, and we describe the details of the proposed cache architecture with the paged cache, and, in Section 3, we give a detailed timing estimation model and area analysis for the proposed architecture. Experimental results are given in Section 4, and Section 5 offers some conclusions. 


\section{Paged Cache Architecture}

\subsection{Conventional Physical Cache}

Suppose that a cache is organized as a collection of $S=C /(B \times A)$ sets, where $A, B$, and $C$ represent associativity, block size and cache size, respectively. In a virtual memory system with page size of $P$ bytes, the virtual-physical mapping only translates the virtual page number (VPN) into the physical page number (PPN), i.e., the page displacement remains invariant across the mapping process. The only condition that we can overlap the cache access with the TLB access completely is $\log _{2}(S)+\log _{2}(B)<=\log _{2}(P)$. If this condition is not satisfied, i.e., $\log _{2}(S)+\log _{2}(B)>\log _{2}(P)$, the cache access must follow the TLB translation. This serial access is illustrated in Figure 1(a), and it is usually comes up in a typical architecture with large cache. For parallel address translation, this condition is such a vital constraint that limits the cache size to be less than $P \times A$ bytes. To increase the cache size (thus reduce the miss ratio), the first approach is to increase the associativity, but it adds hardware complexity and adversely impacts the access time. Rather than increasing the associativity, another approach is to enlarge the page size. For many computer architectures, however, the page size is typically fixed and enlarging it would require substantial changes to both the hardware architecture and software system. In addition, it may lead to more memory fragmentation.

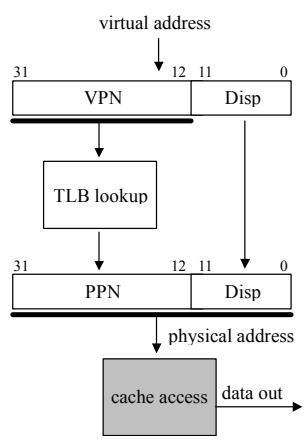

(a) Serial access.

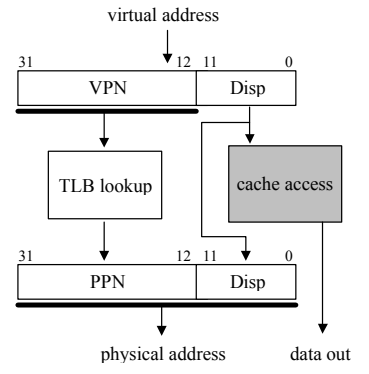

(b) Parallel access.
Figure 1: TLB lookup and cache access flow.

As to the area cost of cache, the cache consists of two arrays used to store the tag and the actual data. The tag array is the storage overhead needed to determine whether the corresponding cached data is what we want. For example, in a $32 \mathrm{~KB} 2$-way cache with block size of 32 bytes, the area proportion of tag array to data array is about 1:15. If the 64-bit wide address space were used, the tag length would be 50 bits and then the area proportion of tag array to data array would be 1:5. From this example, it is clear that the tag area occupies a notable fraction of space as compared to the data area. It will take more area as the memory address space increases.

\subsection{Paged Cache}

We now propose a new cache architecture, called paged cache, for parallel address translation and tag area cost reduction. In the paged cache shown in Figure 2(b), we divide the entire cache into several pieces, named partitions, and limit the partition size to be not larger than the page size. Note that the number of partitions must be the same as the number of TLB entries. In contrast to the conventional cache, each partition is dedicated to only one page that is cached in TLB. There is one-to-one correspondence between partitions and TLB entries. Consequently, we build the strong connection between the cache access and the TLB access, that is, the cache hit implies the TLB hit (or the TLB miss implies cache miss).

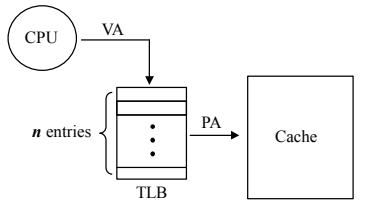

(a) Conventioanl cache. (b) Paged cache.

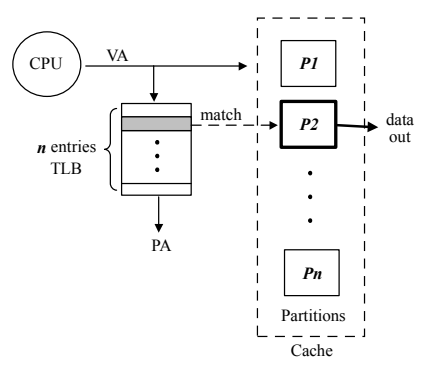

Figure 2: Cache architecture.

By restricting the range in which the cached block can be placed, we can map one page into the specific partition. The tag length in the paged cache is determined by the partition size and given by:

$$
\text { tag length }=\log _{2}\left(\frac{\text { page size }}{\text { partition size }}\right) \text {. }
$$

Because the page size is fixed at $4 \mathrm{~KB}$ throughout this paper, the reasonable partition sizes used in this paper are $1 \mathrm{~KB}, 2 \mathrm{~KB}$ or $4 \mathrm{~KB}$, and thus the tag length are 2-bit, 1-bit or 0-bit, respectively.

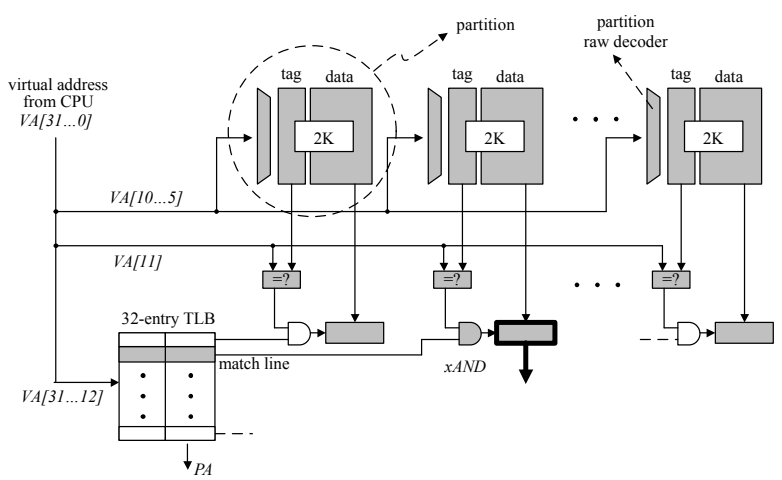

Figure 3: A paged cache example with a 32-entry fullyassociative TLB and a 64KB cache. (The gray blocks symbolize active partition.) 
Consider a $64 \mathrm{~KB}$ paged cache with a 32-entry fullyassociative TLB as shown in Figure 3. Throughout this paper, we fix the address space to be 32-bit wide, the page size $4 \mathrm{~KB}$ and the block size $32 \mathrm{~B}$. Because the number of partitions must equal the number of TLB entries, the size of partition is $2 \mathrm{~KB}$. For mapping one page to one partition, we have to use 1-bit as tag to determine whether the access is hit or not. The address format is shown in Figure 4(b). Compared to the conventional one shown in Figure 4(a), which is a one-way $64 \mathrm{~KB}$ cache architecture, we can observe the tag length is reduced from 16-bit to 1bit. Hence, the tag area can be largely reduced. The access flow in the paged cache architecture is shown in Figure 5 and described as follows:

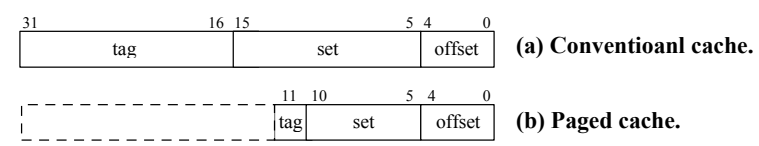

Figure 4: Address format.

1. The virtual address generated by the CPU is concurrently fed into the TLB and the decoder of each partition. The bus widths of input for TLB and partition decoder are 20-bit (i.e., $V A[31 \ldots 12]$ ) and 6-bit (i.e., $V A[10 \ldots 5]$ ), respectively.

2(a). In case of TLB hit, the match lines assert one input of $A N D$ gates that enables/disables the corresponding partition latch to output the accessed data.

2(b). In case of TLB miss, it implies this reference would be a miss in cache access. We must reload the demanded page from the page table. It is important to note that if this reloading induces the TLB replacement, the partition that is corresponding to the replaced page must be flush. By resetting the valid bit of all cache blocks, the partition can be easily flushed.

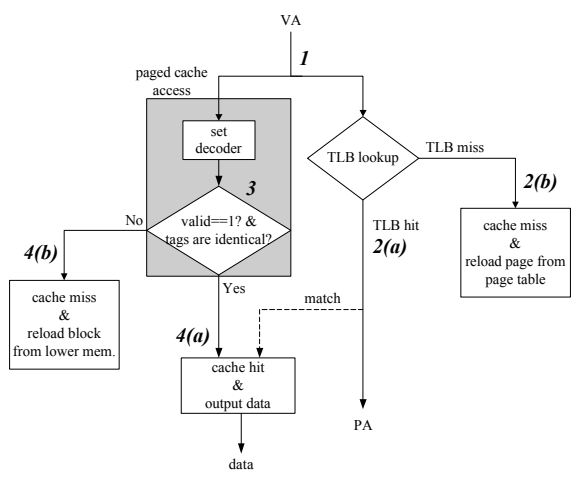

Figure 5: Access flow in the paged cache architecture.

3. To determine a cache hit, we must concurrent check the valid bit and compare the tag with that of selected block. If the valid bit of selected block is 0 , it means this block is not available. 4(a). In case of a cache hit, the hit signal asserts the other input of $A N D$ gate, and the desired data are returned to the processor.

4(b). In case of a cache miss, the physical address generated by the TLB can be used to load the desired block from lower level memory, then the corresponding valid bit and tag must be updated.

\section{Paged Cache Analysis}

\subsection{Tag Area Cost Analysis}

An important cost measure for on-chip cache is its occupied silicon area (or chip area). In this paper, we carry out the simplest area cost for the conventional cache and the paged cache, respectively, using the number of tag cells. The area of additional control circuit used in the paged cache architecture is not considered here due to insignificance, as shown in Figure 3. Because the tag array of paged cache and that of conventional cache have the same structure and implementation, the reduction in terms of number of tag cells in paged cache architecture can directly reflect the actual savings in chip area. Note that the sizes of data array for both architectures are identical.

Table 1: Tag length for various cache configurations.

\begin{tabular}{|c|c|c|c|c|c|c|}
\hline & 1-way & 2-way & 4-way & $\mathrm{P}=1 \mathrm{~K}$ & $\mathrm{P}=2 \mathrm{~K}$ & $\mathrm{P}=4 \mathrm{~K}$ \\
\hline $16 \mathrm{~K}$ & 18 & 19 & 20 & 2 & 1 & 0 \\
\hline $32 \mathrm{~K}$ & 17 & 18 & 19 & 2 & 1 & 0 \\
\hline $64 \mathrm{~K}$ & 16 & 17 & 18 & 2 & 1 & 0 \\
\hline $128 \mathrm{~K}$ & 15 & 16 & 17 & 2 & 1 & 0 \\
\hline
\end{tabular}

Based on the cache configuration, the length of tag for both conventional cache and paged cache is summarized in Table 1. We observed that the tag length of conventional cache is dependent on the cache size and associativity. By contrast, the tag length of paged cache is only dependent on the partition size. For example, suppose that the partition size is $1 \mathrm{~K}(P=1 K)$. Because the page size is $4 \mathrm{~K}$, there are four possible blocks can be mapped to the same set. Consequently, we need two bits as tag to check whether the selected set contains the required data. Because we do not take into account the valid bit, the tag length is 0 for partition with $4 \mathrm{~K}$ size. In Figure 6, we depict the number of tag cells in the caches for the various configurations of the paged cache architecture.

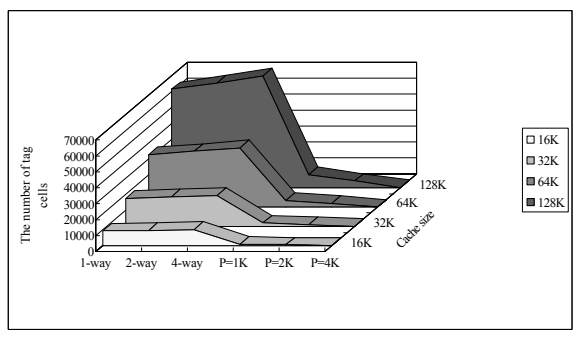

Figure 6: The number of tag cells for various cache configurations. 


\subsection{Extended Access Time}

Up to this point, we have investigated the impact of area cost of the proposed architecture. The other important factor is the cache access time. In this paper, we use the CACTI timing model proposed by Jouppi[7] to estimate the cache access time. In Table 2, we summarize the cache access time for various configurations.

Table 2: Cache access time (ns) for various configurations.

\begin{tabular}{|c|c|c|c|c|c|c|}
\hline & 1-way & 2-way & 4-way & $\mathrm{P}=1 \mathrm{~K}$ & $\mathrm{P}=2 \mathrm{~K}$ & $\mathrm{P}=4 \mathrm{~K}$ \\
\hline $16 \mathrm{~K}$ & 0.99 & 1.37 & 1.47 & 0.67 & 0.71 & 0.76 \\
\hline $32 \mathrm{~K}$ & 1.20 & 1.56 & 1.65 & 0.67 & 0.71 & 0.76 \\
\hline $64 \mathrm{~K}$ & 1.34 & 1.71 & 1.92 & 0.67 & 0.71 & 0.76 \\
\hline $128 \mathrm{~K}$ & 1.66 & 2.02 & 2.07 & 0.67 & 0.71 & 0.76 \\
\hline
\end{tabular}

For a conventional cache, as the degree of cache associativity were varied from 1 to 4 , the increase in access time is as expected. By contrast, the cache access time for our proposed paged cache is only dependent on the partition size, no matter how many partitions are used (i.e., no matter how large the cache size is used). This is because all the partitions in the paged cache are accessed concurrently. Consequently, access time of the paged cache architecture is independent of cache size. The sizeindependent property in cache access time is especially favorable for the high performance processors with a large cache.

Because the TLB can be integrated with the cache memory subsystem, we define the extended cache access time $(E C A T)$ as the time from the arrival of the virtual address generated by $\mathrm{CPU}$ until the required data read from the cache. Note that the ECAT consists of the TLB translation time and cache access time. For example, in a conventional architecture with TLB and cache accessed in serial, ECAT is the sum of TLB translation time and cache access time, i.e., $E C A T_{L 1 C o n v}=T_{T L B}+T_{\text {cache }}$. As we know, for speeding up the address translation, the TLB tag, i.e., virtual page number (VPN) part, is usually implemented with the content addressable memory $(C A M)$. We can split the TLB access into comparison phase and dataout phase. In the comparison phase, we determine whether TLB is hit or not. Once TLB is hit, the data corresponding (i.e., the physical page number $P P N$ ) to the hit entry would be output in the dataout phase.

The CACTI tool described previously is very useful to estimate the cache access time, but it is not suitable to estimate the TLB access time. To measure the TLB access time (i.e., address translation time), we perform the HSPICE timing simulation with a $0.18 \mu \mathrm{m}$ technology and $1.8 \mathrm{~V}$ voltage supply. In this simulation, we use the moderate TLB studied in this paper (i.e., a 64-entry fullyassociative TLB) to validate our proposed paged cache architecture. The HSPICE waveforms of comparison phase and dataout phase are shown in Figure 7(a)(b).

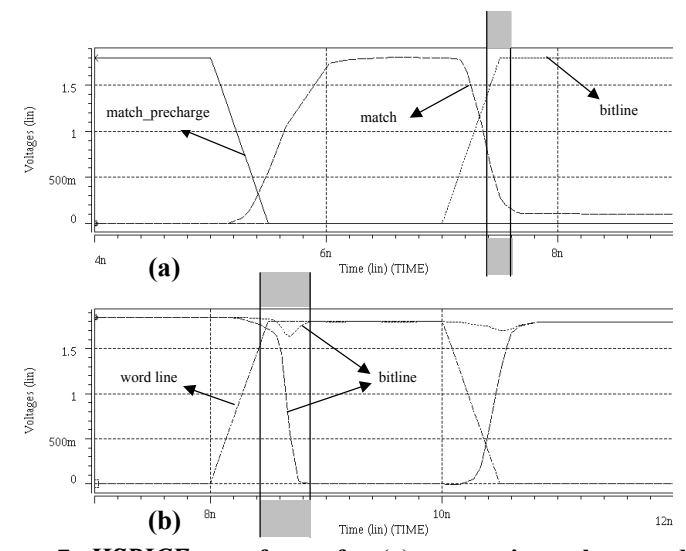

Figure 7: HSPICE waveforms for (a) comparison phase and (b) dataout phase.

As graph shows, the time to determine a TLB hit is about $0.2 n s$, and the time to output the required $P P N$ is about $0.4 n s$. Therefore, the address translation time is $0.6 \mathrm{~ns}$. For the paged cache architecture, because the tag bits must be within the page displacement that remains invariant across the address translation process (as shown in Figure 4), the physical addresses generated by TLB are not necessary in performing the cache access. However, the result of comparison phase in TLB access is critical to overlap cache access with address translation. We must ensure that comparison result is available before the data are outputted from partitions. As illustrated in Figure 8, the TLB comparison time is shorter than the cache access time (as shown in Table 2) whatever partition sizes are used. Consequently, we certified that our proposed architecture can overlap cache access with address translation completely. By the definition of extended cache access time, the ECAT of the proposed paged cache is equal to the access time of one partition, i.e., $E C A T_{L 1_{-} P C}=T_{\text {partition. }}$. Compared to the ECAT of a conventional cache with TLB and cache accessed in serial $\left(E C A T_{L 1_{C} C o n v}=T_{T L B}+T_{\text {cache }}\right)$, the $E C A T_{L 1_{-} C}$ is always shorter than the $\bar{E} C A T_{L 1 \_C o n v}$.

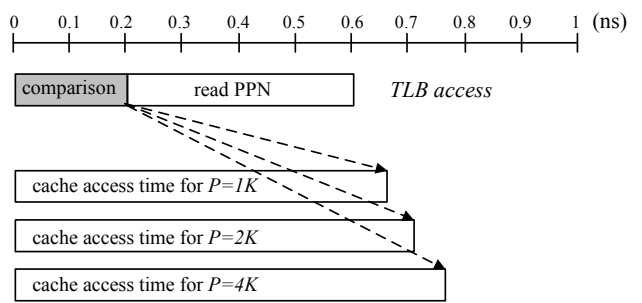

Figure 8: Overlapping cache access with address translation in the paged cache architecture.

\section{Experimental Results}

In this paper, we use SimpleScalar [8] in conjunction with the HSPICE simulations (with a $0.18 \mu \mathrm{m}$ technology 
and $1.8 \mathrm{~V}$ voltage supply) to evaluate the proposed architecture. The numbers of cache accesses were obtained from the simulation of a set of SPEC2000 benchmarks. To get a good mix of CPU-intensive and memory-intensive loads, we randomly chose eight CINT2000 benchmarks (164.gzip, 175.vpr, 176.gcc, 181.mcf, 197.parser, 253.perlbmk, 255.vortex, 256.bzip2) and four CFP2000 benchmarks (177.mesa, 179.art, 183.equake, 188.ammp).

\subsection{Configurations Studied}

For the results presented here, we use a two level onchip cache hierarchy, with split L1 caches and unified L2 cache. To avoid an explosion in the number of results, the page size is fixed as $4 \mathrm{~KB}$, so the partition size can be $1 \mathrm{~KB}, 2 \mathrm{~KB}$ or $4 \mathrm{~KB}$. For our base case, we assume a $64 \mathrm{~KB}$, direct-mapped L1 I-cache and a $64 \mathrm{~KB}$ 4-way L1 D-cache. The block sizes for both caches are set to 32B. The L2 cache is assumed to be a $256 \mathrm{~KB} 4$-way unified on-chip cache with a block size of $64 \mathrm{~B}$.

\subsection{Results and Discussions}

Figure 9 shows the impact of using the paged cache architecture on the hit ratio. In the conventional architecture, the hit ratio increases with the degree of associativity. The decrease in hit ratio with the use of the paged cache architecture is as expected; this is because the paged caches hold only blocks belonging to the pages that are contained in the TLB. An interesting variation in hit ratio is observed between the I-caches and the D-caches. For the I-caches, which has superior spatial locality, increasing the partition size to $4 \mathrm{~KB}$ for the smaller cache (i.e., $16 \mathrm{~K}$ and $32 \mathrm{~K}$ in Figure 9 (a)) does not result in hit ratio improvement as in the case of larger cache (i.e., $64 \mathrm{~K}$ or $128 \mathrm{~K})$. This is because the number of partitions is too small to cache sufficient pages. By contrast, for the Dcaches which has poor locality, decreasing the partition size to $1 \mathrm{~KB}$ (i.e., enlarging the number of partitions) would be beneficial for all cases.

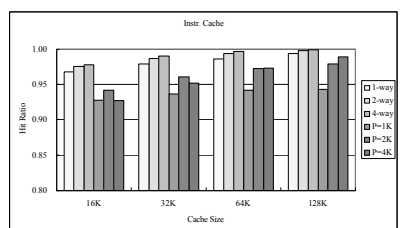

(a) Instruction cache.

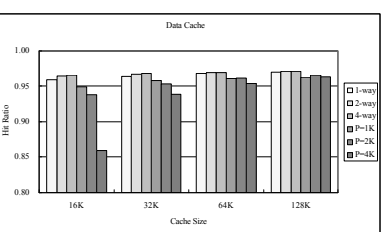

(b) Data cache.
Figure 9: Hit ratio for various conventional caches and paged caches.

In the following discussions, we use tag area cost and extended cache access time as the evaluation criteria to compare the base case implemented in conventional cache architecture with that implemented in paged cache architecture.
Tag Area Cost: In Figure 10, we depict how hit ratio in our base case is reduced by using the paged cache architecture with various partition sizes. From these results, we decide to use the page cache with $4 \mathrm{~KB}$ partition size for I-cache, because the hit ratio decrease is the smallest (i.e., from 0.98 to 0.97 ). Similar to the Dcache, the partition size that we use in the proposed architecture is $1 \mathrm{~KB}$.

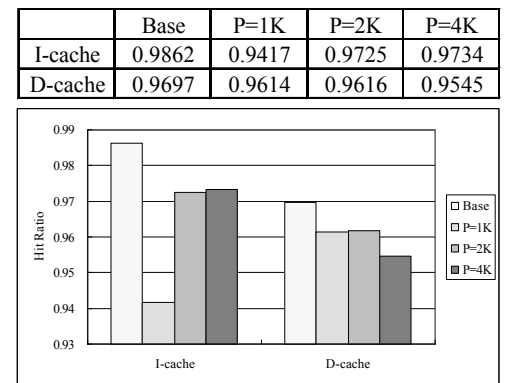

Figure 10: Hit ratio for base case and paged cache architecture.

For simplicity, we use the number of tag cells to estimate the area of tag array in the paged cache architecture. The tag length is 16-bit for the base case of L1 I-cache, and it is 18-bit for the base case of L1 Dcache. Because the partition size is $4 \mathrm{~KB}$, the tag length is 0 -bit for the paged L1 I-cache. In the paged L1 D-cache, the tag length is 2-bit. Note that we do not take into account the status bits, such as valid bit, dirty bit, etc. For a $64 \mathrm{~KB}$ cache, there are $2^{11}=2048$ blocks in the data array. As we know, each cache block in the data area has only one corresponding tag. For the L1 I-cache, the number of tag cells for the conventional cache and the paged cache are $2^{11} \times 16$ and 0 , respectively, i.e., the tag area savings is $100 \%$. In the case of L1 D-cache, the number of tag cells was reduced from $2^{11} \times 18$ to $2^{11} \times 2$, representing the tag area savings of about $88 \%$.

Extended Cache Access Time: A simple rule of hardware design: Smaller is faster. This simple principle is particularly applicable to memories built from the same technology. Because the partition in our proposed architecture is smaller than the conventional L1 cache, it generally has a faster access time. We use the CACTI tool, described in [7], to estimate the access time of the conventional on-chip caches, as well as the paged cache that was proposed to reduce the tag area cost dramatically. Note that the cache models used in this tool is not precisely equal to the paged cache. Actually, the access time of the partition is slightly faster than the results shown here. This is because the tag length of our proposed architecture is far shorter than that of the conventional cache. In our experiments, the partition in the paged cache is implemented as a direct-mapped organization, with size ranging from $1 \mathrm{~KB}$ to $4 \mathrm{~KB}$, and block size is 32 bytes. 
Table 2 shows the cache access time of the base case and the paged cache with various partition sizes.

In this study, for investigating the impact of address translation delay, we use the extended cache access time (ECAT, defined in Section 3.2) instead of the cache access time. Based on the memory hierarchical model, the average extended cache access time for the conventional cache and our proposed architecture can be expressed by the following equations:

$$
\begin{aligned}
& E C A T_{\text {Conv_ave }}=E C A T_{L 1 \_C o n v}+\left(1-H R_{L 1 \_C o n v}\right) \times T_{L 2}, \\
& E C A T_{P C \_ \text {ave }}=E C A T_{L 1 \_P C}+\left(1-H R_{L 1 \_P C}\right) \times T_{L 2},
\end{aligned}
$$

where $E C A T_{L 1 C o n v}$ and $E C A T_{L 1 P C}$ are the extended access time of the $\mathrm{L} 1$ conventional cache and $\mathrm{L} 1$ paged cache, respectively, $H R_{L 1}$ is the hit ratio of the $\mathrm{L} 1$ cache, and $T_{L 2}$ is the access time of the L2 on-chip cache. According to the definition of ECAT, ECAT $T_{L 1 C o n v}$ is the sum of TLB translation time and cache access time, and $E C A T_{L 1 P C}$ is equal to the access time of partition, because our proposed architecture can completely overlap cache access with address translation, as described in Section 3.2.

Combine Equations (1), (2) and the results illustrated in Table 3(a), the average extended cache access time measured in nanosecond for both conventional cache and paged cache are shown in Table 3(b). The key observation is that approximately a $58 \%$ reduction in average $E C A T$ when using the paged L1 I-cache with $4 \mathrm{~KB}$ partition size, and $70 \%$ for the paged L1 D-cache with $1 \mathrm{~KB}$ partition size can be achieved. Consequently, our proposed cache architecture can improve the extended cache access time, and this improvement may present an opportunity to increase the fundamental processor clock.

\begin{tabular}{|c|c|c|c|c|c|c|}
\hline & $E C A T_{L 1 \text { Conv }}$ & $E C A T_{L 1 P C}$ & $T_{L 2}$ & & $E C A T_{\text {Conv ave }}$ & $E C A T_{P C \text { ave }}$ \\
\hline & & \begin{tabular}{|l|}
0.7649 \\
\end{tabular} & \multirow{2}{*}{2.6383} & & & \\
\hline cache & 2.5237 & 0.6749 & & D-cache & 2.6037 & 0.776 \\
\hline
\end{tabular}

Table3: Extended cache access time (in $n s$ ).

(a) Extended cache access time of $L 1$ cache. $\left(T_{L 2}\right.$ is the access time of $L 2$ cache.)

(b) Average extended cache access time for both conventional cache and paged cache.

Summarize the performance comparisons in tag area cost and $E C A T$, the improvements due to the use of paged cache are shown in Table 4 . One can see that the paged cache is very effective in reducing area cost for the onchip L1 caches, while the average extended cache access time can also be improved.

Tabel 4: Summary of performance improvement.

\begin{tabular}{|l|c|c|}
\hline & Area Cost & ECAT \\
\hline L1 I-cache & $100 \%$ & $58 \%$ \\
\hline L1 D-cache & $88 \%$ & $70 \%$ \\
\hline
\end{tabular}

\section{Conclusions}

On-chip caches is a major source of area consuming in modern advanced processors. The major area consuming in the conventional cache design is the tag array which is the storage overhead needed to determine whether a reference is hit or not. Besides area cost, address translation is a vital mechanism in modern processors. For parallel accessing of cache and TLB in a processor with physical cache, is the cache size restricted by the product of the page size and the cache associativity? In this paper, a new cache architecture, called paged cache, was proposed, in which we use a simple partition scheme to divide a large cache into several partitions. By restricting the range in which the cached block can be placed, we can eliminate the total or partial tag depending on the partition size. On the other side, because all the smaller partitions are concurrently accessed without waiting for the generation of physical address, the address translation time can be eliminated entirely. The experimental results validated our proposed cache architecture and showed that it is very effective in reducing both tag area cost and extended cache access time in the on-chip L1 caches. For the base case of L1 on-chip I-cache $(64 \mathrm{~KB}, 1$-way), the paged cache with partition size of $4 \mathrm{~KB}$ can result in roughly $100 \%$ reduction in tag area and $58 \%$ reduction in average extended cache access time $(E C A T)$. Similarly, for the base case of L1 on-chip D-cache (64KB, 4-way), the paged cache with partition size of $1 \mathrm{~KB}$ can result in roughly $88 \%$ reduction in tag area and $70 \%$ reduction in average $E C A T$.

\section{References}

[1] A. J. Smith, "Cache Memories," Computing Surveys, Vol. 14, No. 3, September 1982, pp. 473-530

[2] T. Chiueh and R. Katz, "Eliminating the Address Translation Bottleneck for Physical Address Cache," in Proc. 5th ASPLOS, Sept. 1992, pp. 137-148.

[3] K. Inoue, H. Nonogaki, Y. Urakawa and K. Shimizu, "Plural Virtual Address Space Processing System," U.S. Patent No. 4145738, 20 Mar. 1997.

[4] K. Hua et al., "Early Resolution of Address Translation in Cache Design," Proc. Int'l Conf. Computer Designs, Oct. 1990, pp. 408-412.

[5] T. Austin and G. Sohi, "High-Bandwidth Address Translation for Multiple-Issue Processors," in Proc. of 23rd International Symposium on Computer Architecture, May 1996, pp. 158-167.

[6] G. Taylor, P. Davis and M. Farmwald, "The TLB Slice A Low-Cost High-Speed Address Translation Mechanism," in Proc. of 17th International Symposium on Computer Architecture, May 1990, pp. 355-363.

[7] S. E. Wilton and N. Jouppi, "An Enhanced Access and Cycle Time Model for On-Chip Caches," DEC WRL Research Report 93/5, July 1994.

[8] D.C. Burger and T. M. Austin, "The SimpleScalar Tool Set, Version 2.0," Computer Architecture News, 25 (3), pp. 13-25, June, 1997. Extended version appears as UW Computer Sciences Technical Report \#1342, June 1997. 